\title{
Effects of lidocaine administration using a newly developed urethral catheter on catheter removal pain: A randomized clinical trial
}

\author{
Kazuhiro Kubo ${ }^{1}$, Tomonori Takazawa ${ }^{2 *}$, Tomonobu Sato ${ }^{1}$, Tatsuo Horiuchi ${ }^{1}$ and Shigeru Saito ${ }^{1}$ \\ ${ }^{1}$ Department of Anesthesiology, Gunma University Graduate School of Medicine, Japan \\ ${ }^{2}$ Intensive Care Unit, Gunma University Hospital, Japan
}

\begin{abstract}
Objective: To evaluate the safety and efficacy of a urethral catheter designed to administer lidocaine to the urethra in reducing patient pain during catheter removal.

Methods: This was a prospective, randomized study involving 20-59-year old men scheduled to undergo surgery under general anesthesia with urethral catheter removal within 24 hours postoperatively. The catheter was a modified Foley catheter $(14 \mathrm{~F})$ with a temperature sensor, which was removed, in which two holes were created proximal to the balloon. The catheter was inserted into the bladder in the usual manner. The patients were divided into two groups: the lidocaine and control groups. In the lidocaine group, $5 \mathrm{ml}$ of $4 \%$ lidocaine was administered through the holes created in the urethral catheter 10 minutes before catheter removal, and the urinary meatus was compressed with gauze or other material to minimize leakage of the drug solution. Nothing was administered in the control group. The catheter was removed and visual analog scale (VAS) scores of removal pain were assessed by a single evaluator and compared between the two groups.
\end{abstract}

Results: Forty patients were evaluated. There were no significant differences in patient characteristics between the groups. VAS scores at the time of catheter removal were lower in the lidocaine group (median: 11) than in the control group (median: 39) $(\mathrm{P}<0.001$, Mann-Whitney $U$ test). No adverse events were seen in either group.

Conclusion: Use of our modified urethral catheter facilitates local anesthetic administration into the urethra, reducing catheter removal pain.

\section{Introduction}

Urethral catheters are widely used in various settings, such as the perioperative period, in patients who are unable to urinate on their own. Stimulation of the urethra and bladder mucosa that occurs due to catheterization is accompanied by discomfort, a feeling of residual urine, and pain [1]. Furthermore, strong discomfort and pain can also occur during catheter removal. Lidocaine, a local anesthetic, has been widely used by urologists for cystoscope insertion for many years, due to its well-known analgesic effects during the procedure $[2,3]$. We decided to use lidocaine to alleviate the pain that occurs during urethral catheter removal. However, a device that allowed the administration of a local anesthetic into the urethra did not previously exist. In the present study, we therefore developed a catheter that is structured such that it can administer lidocaine into the urethra. To achieve this, we created two holes in a ready-made catheter to inject the drug solution. In the present study, to assess the effectiveness of this newly developed catheter, patient pain during catheter removal was evaluated using a visual analogue scale (VAS). The safety of using this catheter was also examined.

\section{Methods}

This study used a prospective, randomized design, and was registered with the UMIN registry (UMIN-CTR ID: 000015289). Informed consent was obtained from all patients. The study subjects were 20-59year old men who were scheduled to undergo surgery under general anesthesia [American Society of Anesthesiologists (ASA) physical status $[1,2]]$ and were also scheduled to undergo urethral catheter removal within the first 24 hours after surgery. Detailed inclusion and exclusion criteria are shown in Table 1. Only men were included in this study, since, compared to women, men have a longer urethra, which usually results in greater catheter-related discomfort in them. Patients who were scheduled to receive epidural catheter placement or continuous fentanyl administration for postoperative pain relief were excluded, since these treatments may affect the results of pain assessment; however, the administration of all other postoperative analgesics was allowed in both groups. Considering the effects of drug solution injection on the surgical site, patients who were scheduled to undergo urinary tract surgery and patients diagnosed with inflammation of the urinary tract were excluded. In addition, patients with arrhythmia and/or on antiarrhythmic medication, including amiodarone, were excluded, to avoid cardiac hypofunction due to lidocaine administration. Although the anesthesia protocol was determined by the attending anesthesiologist, combination of sevoflurane or desflurane with remifentanil was used for maintenance of general anesthesia in most cases.

Patients who met the subject criteria were identified at the preoperative examination, and their consent was subsequently obtained. With reference to previous studies, we assumed that the visual analog

*Correspondence to: Tomonori Takazawa, Intensive Care Unit, Gunma University Hospital, 3-39-15 Showa-machi, Maebashi 371-8511, Japan, Tel: +8127-220-8698, Fax: +81-27-220-8692, E-mail: takazawt@gunma-u.ac.jp

Key words: urinary catheterization, pain, lidocaine

Received: June 05, 2018; Accepted: June 19, 2018; Published: June 22, 2018 
Table 1. Inclusion and exclusion criteria in the study

\section{Inclusion criteria}

- Male between 20 and 60 years of age

- Urethral catheter (14F) planned for the perioperative period

- ASA Physical Status (PS) classification 1or 2

- General anesthesia without epidural anesthesia or intravenous patient-controlled analgesia (IVPCA) is planned

\section{Exclusion criteria}

- Urinary tract procedure

- Past history of hypersensitivity to lidocaine or amide type local anesthetics

- Arrhythmia on ECG or on anti-arrhythmic medication

- Disturbed consciousness or difficulty understanding

scale (VAS) score would decrease by 20 in the lidocaine group [4], and the standard deviation of VAS was estimated to be 20 [5]. Based on these estimations, the required sample size was determined to be 17 patients with power analysis using $\alpha=0.05$ and $\beta=0.8$. Assuming a $10 \%$ dropout rate, the plan was to enroll 20 participants per group. Patients who gave consent to participate were randomized and enrolled into two groups using specific computer software. Based on the randomization, patients either received lidocaine (Astra Zeneca Co. Ltd, London, United Kingdom) ("lidocaine group") or did not receive lidocaine ("control group") via the urethral catheter, and comparisons were made between the two groups. Since the analgesic effects of lidocaine administration in the urethra have already been amply shown, the control group did not receive any solution, including saline, through lumen $\mathrm{C}$, i.e. we merely compared pain on catheter removal with and without administration of lidocaine. Hence, this study focused on the ability of the novel device as a tool for drug administration into the urethra, and not on the pharmacological effects of lidocaine.

The primary endpoint was the VAS score at the time of catheter removal. The VAS is a straight line with 0 and 100 marked at the ends and no marks in between, on which the patient is asked to indicate the degree of pain with a pen $(0=$ no pain, $100=$ unbearable pain $)$.

\section{Catheter creation}

The catheter used in this study was created by modifying a Foley catheter $(14 \mathrm{~F})$ with a temperature sensor (NOVATEMP Foley Catheter, NOVAMED-USA, New York, USA). The thermometer part was extracted, and two holes were created, one at $2 \mathrm{~cm} \mathrm{(A)} \mathrm{and} \mathrm{the} \mathrm{other}$ at $5 \mathrm{~cm}$ (B) proximal to the urethral catheter balloon, to serve as drug solution openings (Figure 1). The holes were created in lumen $\mathrm{C}$, which was originally made as a lumen for inserting a temperature sensor, using a $22-\mathrm{G}$ needle. This procedure was conducted in an operating room under a clean environment, and outflow from the opening was verified by injecting saline. Anatomically, the urethral sphincter is 3 to $4 \mathrm{~cm}$ from the neck of the bladder in men. In patients with prostatic hyperplasia, the position of the urethral sphincter may be displaced; thus, the opening would need to be created further distally. Hence, in the catheter we developed, to achieve drug action in the areas of the bladder neck and urethral sphincter, the openings for drug solution injection were positioned $2 \mathrm{~cm}$ and $5 \mathrm{~cm}$ from the neck of the bladder. Further, older men ( $\geq 60$ years old) have an increased prevalence of prostatic hyperplasia and were, therefore, excluded from this study. To connect the syringe for drug injection to lumen $\mathrm{C}$, the drug injection port, Safe Access (Covidien, Dublin, Ireland) was inserted at the proximal end of lumen $\mathrm{C}$. After induction of general anesthesia but before the operation, the catheter was inserted into the bladder, and the balloon was inflated and fixed with $10 \mathrm{ml}$ of distilled water.

\section{Timeline of lidocaine administration and assessment of pain}

In the lidocaine group, $5 \mathrm{ml}$ of $4 \%$ lidocaine was administered through lumen $\mathrm{C}$ of the urethral catheter (Figure 1) 10 minutes before catheter removal. The lidocaine dose was determined based on treatments and procedures performed at the urology department. Procedures such as cystoscopy are started 10 minutes after injecting an appropriate amount of $2-4 \%$ lidocaine [2]. Since a substantial amount of drug solution can leak from the urinary meatus after injection, a relatively high concentration ( $5 \mathrm{ml}$ of $4 \%$ lidocaine solution containing $200 \mathrm{mg}$ of lidocaine) was used in this study. Lidocaine administration was performed over 3 minutes to minimize the discomfort and/or pain associated with its injection. After lidocaine administration, the urinary meatus was compressed for 3 minutes with gauze or other material to minimize leakage of the drug solution. The purpose of this intervention was to allow contact time for the drug to act more effectively. Four minutes later, the catheter balloon was deflated by removal of the distilled water. Subsequently, the catheter was removed, and the patient's VAS score for catheter removal pain was assessed by a single evaluator. The catheter was similarly removed in the control group, although without administration of any kind of solution, and VAS scores were compared between the two groups.

\section{Statistical analysis}

All data were expressed as mean \pm S.D. The choice of statistical technique depended on the results of equal variance tests. The unpaired $t$ test was used for comparisons of patient age, height and weight. Mann-Whitney $U$ test was used for comparison of VAS scores and duration of catheterization between the two groups. Fisher's exact test was performed to compare the incidence of patients with diabetes mellitus in each group. $\mathrm{P}<0.05$ was considered as significant.

A
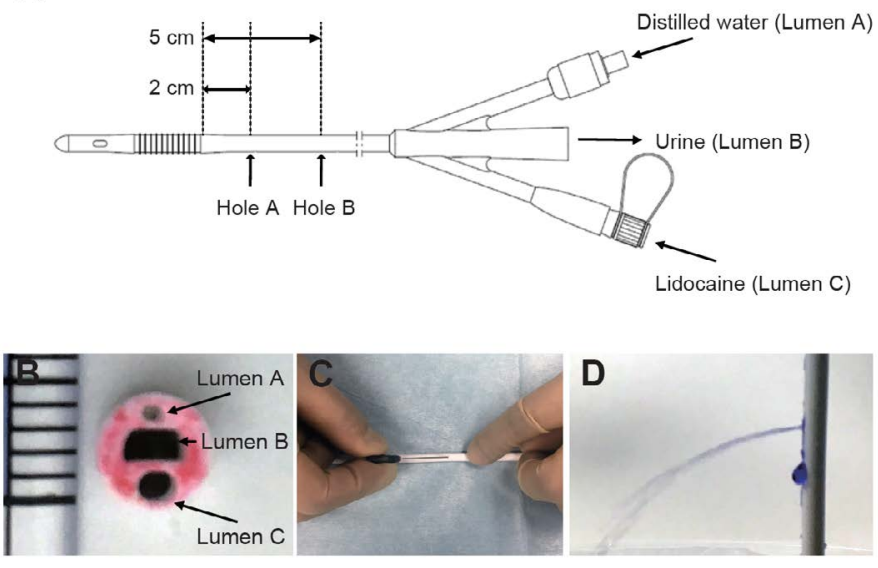

D

Figure 1. Schema of the newly developed urethral catheter and pictures of drilling holes process.

A shows an outline of the catheter. The catheter has three lumens. Lumen A is connected to balloon. Distilled water is administered through this lumen for balloon dilation. Lumen B is connected to urine collection bag. Lumen $\mathrm{C}$ is used to administer lidocaine. The lumen has hole A and B that is located at 2 and $5 \mathrm{~cm}$ from proximal edge of the balloon. Figure B is a picture of the cross section of the catheter. To emphasize the holes, the cross section was colored with red ink. One increment indicates $1 \mathrm{~mm}$. Figure $\mathrm{C}$ shows the process of making holes in the catheter. The holes were made from the outside of the catheter by puncturing a 22-G needle towards Lumen C. Normal saline was injected into Lumen C to confirm that the holes were open and that no other parts were broken. In order to make the outflow of normal saline easier to see for figure only, crystal violet was used as a dye (D). These processes were done with sterile gloves on a sterile cloth in an operating room 


\section{Results}

Forty patients were evaluated between July 2011 and March 2012. Figure 2 shows the flow chart of performance of this study. The surgeries that patients underwent were as follows: 16 orthopedic, 11 otolaryngologic, 8 oral, 4 general, and 1 dermatologic surgery. There were no significant differences between the two groups in patient characteristics, except for height (Table 2). The number of patients with diabetes mellitus was assessed, because they may have hypesthesia due to peripheral neuropathy, which in turn can influence VAS scores. Only one patient in the control group was diabetic (Table 2). There were no dropouts throughout the study, and the catheter was removed, and subjects were evaluated within 24 hours after surgery in most cases. Induction and maintenance of anesthesia and awakening from anesthesia were achieved without complications in all patients. There were no issues with the level of consciousness during assessment in any of the patients, and VAS scores were appropriately assessed. The median value of VAS score in the lidocaine group was 11 (range: $0-32$ ) While, those in the control group was 39 (range: 6-92). These results indicated significantly lower VAS scores at the time of catheter removal in the lidocaine group (Mann-Whitney $U$ test, $\mathrm{P}<0.001$, Figure 3 ). Drug solution leakage from the urinary meatus was verified in all patients in the lidocaine group. Upon urethral catheter removal, adverse events, such as bleeding from the external urethral meatus, were not observed in any of the patients. In addition, abnormal vital signs and abnormalities in the urinary system, such as urinary retention and infection, were not observed after catheter removal in either group.

\section{Discussion}

The purpose of this study was to develop a method to alleviate the pain that arises during urethral catheter removal by injecting a local

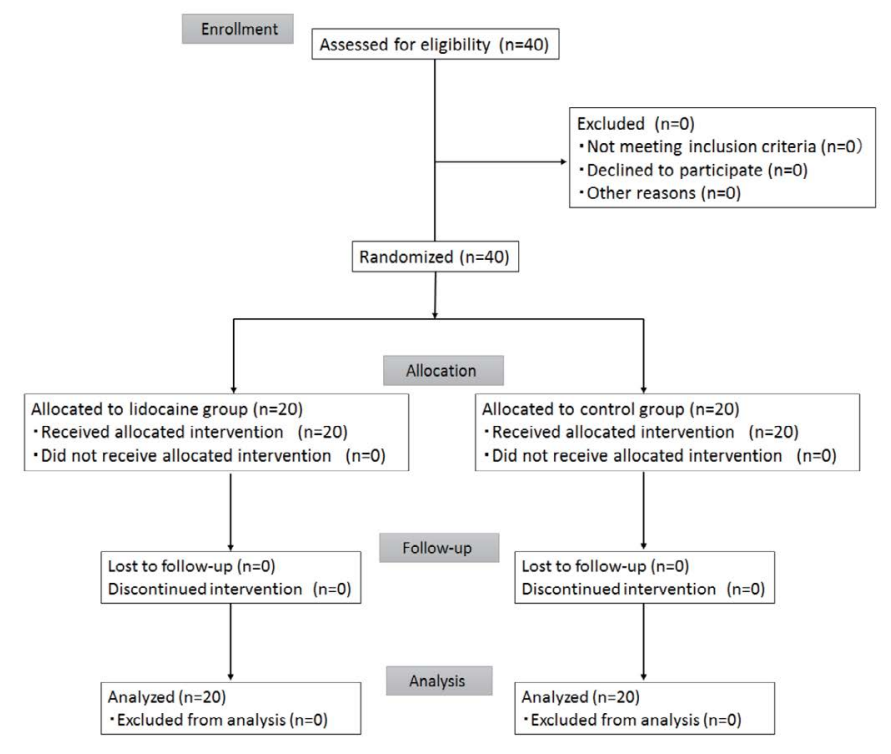

Figure 2. Flow chart of the study

Table 2. Patient characteristics and duration of catheterization in the two groups

\begin{tabular}{|l|c|c|}
\hline & Control (n=20) & Lidocaine (n=20) \\
\hline Age $(\mathrm{yr})$ & $36.7 \pm 11.8$ & $41.1 \pm 12.2$ \\
\hline Height $(\mathrm{cm})$ & $171.1 \pm 8.1$ & $166.0 \pm 5.9$ \\
\hline Weight $(\mathrm{kg})$ & $69.5 \pm 12.7$ & $63.5 \pm 12.4$ \\
\hline Duration of catheterization $(\mathrm{h})$ & $20.4 \pm 2.6$ & $19.2 \pm 2.8$ \\
\hline Number of diabetic patient & 1 & 0 \\
\hline
\end{tabular}

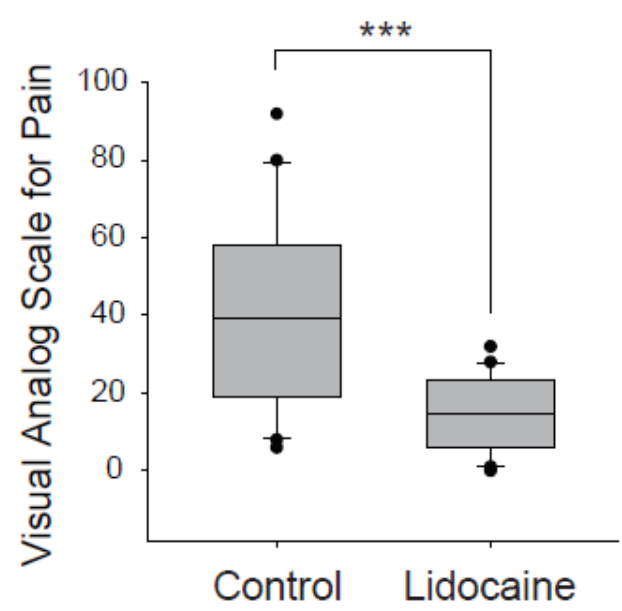

Figure 3. Comparison of visual analog scale for pain at the time of catheter removal between control and lidocaine group. ${ }^{* *} \mathrm{P}<0.001$, Mann-Whitney $\mathrm{U}$ test. The boundary of the box closest to zero indicates the $25^{\text {th }}$ percentile, a line within the box marks the median, and the boundary of the box farthest from zero indicates the $75^{\text {th }}$ percentile. Whiskers (error bars) above and below the box indicate the $90^{\text {th }}$ and $10^{\text {th }}$ percentiles

anesthetic via a newly developed catheter, and to assess its effects. With our method, it was possible to significantly reduce pain in the lidocaine group compared to the control group. Moreover, no side effects associated with local anesthetic usage were observed.

Although the objective of this study was to develop a method to alleviate the pain that arises during urethral catheter removal, we believe that the catheter can be used for other purposes as well. One such application is to reduce or prevent catheter-related bladder discomfort (CRBD). The incidence of CRBD in surgeries of the lower urethra was reported to be $50-90 \%$ in a previous study [6], and it is therefore a significant issue in patients who undergo urethral catheterization.

The internal urethral sphincter, from the neck of the bladder to the prostate, is an involuntary muscle, and muscarinic receptors (M2, M3) regulate its contractions [7]. It has been previously reported that the incidence and severity of CRBD can be decreased by injecting anticholinergic agents, such as tolterodine [8], oxybutynin [8,9], and butylscopolamine [10], locally in the urethra. Moreover, analgesics such as tramadol [11], ketamine [12], paracetamol [6], and pregabalin [13] have also been reported to be effective in the prevention and treatment of CRBD. However, these drugs must be administered systemically via intravenous catheters and are also known to induce side effects, such as dry mouth, flushing of the face, blurred vision, sedation, nausea, and vomiting $[6,8-13]$. By using the catheter that we developed, local administration of not only local anesthetics, but also various other analgesic agents, to the urethral mucosa becomes feasible; thus, it is anticipated that this device will enable the prevention of CRBD, while simultaneously minimizing the side effects of the drugs injected.

This study has certain limitations. We believe that the decrease in VAS scores at catheter removal with lidocaine administration was due to the anesthetic effects of lidocaine. However, since there was no placebo group with saline injection, we cannot rule out the possibility that the pain relief during catheter removal may have been achieved by separation of the catheter from the urethra by lidocaine injected prior to catheter removal. Moreover, both the patients and the person assessing the VAS were not blind to which intervention had been performed. Double blind tests using saline as a control group will be necessary in the future. 


\section{Conclusion}

We developed a new urethral catheter with strategically positioned holes in it to administer drug solutions. We showed that lidocaine administration into the urethra via this device just before catheter removal could reduce patient pain during catheter removal without any side effects.

\section{References}

1. Wilde MH (2002) Understanding urinary catheter problems from the patient's point of view. Home Healthc Nurse 20: 449-455. [Crossref]

2. Brekkan E, Ehrnebo M, Malmstrom PU, Norlen BJ, Wirbrant A (1991) A controlled study of low and high volume anesthetic jelly as a lubricant and pain reliever during cystoscopy. J Urol 146: 24-27. [Crossref]

3. Holmes M, Stewart J, Rice M (2001) Flexible cystoscopy: is the volume and content of the urethral gel critical? J Endourol 15: 855-858. [Crossref]

4. Todd KH, Funk KG, Funk JP, Bonacci R (1996) Clinical significance of reported changes in pain severity. Ann Emerg Med 27: 485-489. [Crossref]

5. Kanai A, Kumaki C, Niki Y, Suzuki A, Tazawa T, et al. (2009) Efficacy of a metereddose $8 \%$ lidocaine pump spray for patients with post-herpetic neuralgia. Pain Med 10: 902-909. [Crossref]

6. Ergenoglu P, Akin S, Yalcin Cok O, Eker E, Kuzgunbay B, et al. (2012) Effect of intraoperative paracetamol on catheter-related bladder discomfort: a prospective, randomized, double-blind study. Curr Ther Res Clin Exp 73: 186-194. [Crossref]
7. Yoshimura N, Kaiho Y, Miyazato M, Yunoki T, Tai C, et al. (2008) Therapeutic receptor targets for lower urinary tract dysfunction. Naunyn Schmiedebergs Arch Pharmacol 377: 437-448. [Crossref]

8. Agarwal A, Dhiraaj S, Singhal V, Kapoor R, Tandon M (2006) Comparison of efficacy of oxybutynin and tolterodine for prevention of catheter related bladder discomfort: a prospective, randomized, placebo-controlled, double-blind study. Br J Anaesth 96: 377-380. [Crossref]

9. Tauzin-Fin P, Sesay M, Svartz L, Krol-Houdek MC, Maurette P (2007) Sublingual oxybutynin reduces postoperative pain related to indwelling bladder catheter after radical retropubic prostatectomy. Br J Anaesth 99: 572-575. [Crossref]

10. Ryu JH, Hwang JW, Lee JW, Seo JH, Park HP, et al. (2013) Efficacy of butylscopolamine for the treatment of catheter-related bladder discomfort: a prospective, randomized, placebo-controlled, double-blind study. Br J Anaesth 111: 932-937. [Crossref]

11. Agarwal A, Yadav G, Gupta D, Singh PK, Singh U (2008) Evaluation of intraoperative tramadol for prevention of catheter-related bladder discomfort: a prospective, randomized, double-blind study. Br J Anaesth 101: 506-510. [Crossref]

12. Agarwal A, Gupta D, Kumar M, Dhiraaj S, Tandon M, et al. (2006) Ketamine for treatment of catheter related bladder discomfort: a prospective, randomized, placebo controlled and double-blind study. Br J Anaesth 96: 587-589. [Crossref]

13. Srivastava VK, Agrawal S, Kadiyala VN, Ahmed M, Sharma S, et al. (2015) The efficacy of pregabalin for prevention of catheter-related bladder discomfort: a prospective, randomized, placebo-controlled double-blind study. J Anesth 29: 212-216. [Crossref]

Copyright: (C2018 Kubo K. This is an open-access article distributed under the terms of the Creative Commons Attribution License, which permits unrestricted use, distribution, and reproduction in any medium, provided the original author and source are credited. 